\title{
Performance Analysis of the Intelligent Mobility Optimization CRRM Approach Using a Markovian Chain Model
}

\author{
Abdallah AL Sabbagh, Robin Braun, and Mehran Abolhasan \\ Centre for Real-Time Information Networks (CRIN), University of Technology, Sydney (UTS), Sydney, Australia \\ E-mail: \{abdallah.alsabbagh, robin.braun, mehran.abolhasan\}@uts.edu.au
}

\begin{abstract}
Due to the increasing demand of wireless services, mobile technology has rapidly progressed towards the fourth generation (4G) networking paradigm. This generation will be heterogeneous in nature and it can be achieved through the integration of different Radio Access Technologies (RATs) over a common platform. Common Radio Resource Management (CRRM) was proposed to manage radio resource utilization in heterogeneous wireless networks and to provide required Quality of Service (QoS) for allocated calls. RAT selection algorithms are an integral part of the CRRM algorithms. Their role is to decide, when a new or Vertical Handover (VHO) call is requested, which of the available RATs is most suitable to fit the need of the incoming call and when to admit them. This paper extends our earlier work on the proposed intelligent hybrid mobility optimization RAT selection approach which allocates users in high mobility to the most suitable RAT and proposes an analytical presentation of the proposed approach in a multidimensional Markov chain model. A comparison for the performance of centralized load-balancing, distributed and the proposed intelligent mobility optimization algorithms is presented in terms of new calls blocking probability, VHO calls dropping probability, users' satisfactions probability, average networks load and average system throughput. Simulation and analytical results show that the proposed algorithm performs better than the centralized loadbalancing and distributed algorithms.
\end{abstract}

Index Terms-Next Generation Wireless Network (NGWN); Heterogeneous Wireless Network; 4G; Markov Chain Model; Common Radio Resource Management (CRRM); Radio Access Technology (RAT) Selection Algorithms; Mobility Optimization

\section{INTRODUCTION}

Next Generation Wireless Networks (NGWN) are predicted to be heterogeneous in nature and to provide high speed network connections for a variety of services such as Voice over IP (VoIP), web browsing, File Transfer Protocol (FTP), video streaming, online gaming, real time video and push-to-talk [1] and will interconnect different Radio Access Technology (RATs) such as:

This article is an extended version of the paper titled: "A Mobility Optimization CRRM Approach for Next Generation Wireless Networks" by A. AL Sabbagh, R. Braun and M. Abolhasan which appeared in the International Conference on Computer \& Information Sciences (ICCIS 2012), Kuala Lumpur, Malaysia, June 12-14, 2012.
UMTS Terrestrial Radio Access Network (UTRAN), Long Term Evolution (LTE) and Wireless Local Area Network (WLAN) through a common platform [2-4]. To integrate various wireless network standards under a common platform, a number of key research issues needs to be addressed. One such issue is on how to allocate each user to the most suitable RAT. An efficient solution to this question can lead to maximization of radio resources utilization, better performance for service providers and provision and guarantee the required Quality of Service (QoS) with low cost to users. Currently, Radio Resource Management (RRM) including admission control, congestion control, power control, packet scheduling, horizontal handover (HO), initial RAT selection algorithm and vertical HO (VHO) algorithms are implemented efficiently for the specific RAT it was developed for. However, they are not suitable for heterogeneous wireless networking scenarios where various types of RATs exist. Common Radio Resource Management (CRRM) was proposed to manage radio resource utilization in heterogeneous wireless networks [5].

A number of different CRRM algorithms have been proposed in the literature for heterogeneous wireless networks. CRRM algorithms can be categorized into centralized such as load-balancing algorithm and policy based algorithm or distributed algorithms such as service based algorithm $[6,7]$.

Centralized RAT selection algorithms have the benefit of considering more criteria during the decision making process. However, centralized algorithms do not guarantee required QoS for all admitted calls. In addition, they reduce network capacity as a result of the introduced signaling overheads or delay resulted by the communication between the network entities.

On the other hand, distributed algorithms have the benefit of considering users' preferences. A number of different distributed algorithms are proposed in [8-10]. These algorithms allow User Terminal (UT) to select the most efficient RAT that maximizes its satisfaction which is based on its preference such as best QoS or cheapest cost. However, distributed algorithms do not take into account the network benefits and policies. This may lead to inefficient radio resource utilization and it may create network bottlenecks. 
All the proposed algorithms have limitations and do not provide a complete solution for the RAT selection problem. Therefore there is a need for a hybrid RAT selection algorithm (distributed with network assistance) which will provide the UTs some information that assists them during their decision making process to select the most efficient RAT that maximizes their satisfactions and at the same time improve the efficient radio resource utilization. The IEEE P1900 Standards Committee proposes an IEEE P1900.4 Protocol [11] that is able to provide the required support to the hybrid approach.

In this paper, we extend our earlier work on the proposed intelligent hybrid RAT selection approach for mobility optimization [12, 13] which uses the IEEE P1900.4 Protocol to enable communication between the UTs and the different wireless networks and propose an analytical presentation of this approach in a multidimensional Markov chain model. The proposed approach aims to improve the RRM in heterogeneous wireless networks. This improvement will lead to enhanced QoS for end user, increase users' satisfactions and improve the efficiently of radio resource utilization.

The remainder of the paper is organized as follows. Section II presents the proposed intelligent mobility optimization algorithm. In Section III, the cost function model for the proposed approach is presented. The proposed mobility optimization algorithm is simulated and compared with the centralized load-balancing and distributed algorithms in Section IV. The three algorithms are simulated in a scenario which UTRAN, LTE and WLAN are overlapped in the same geographical area and they are evaluated in terms of new call blocking probability, VHO calls dropping probability and users' satisfactions probability. In Section $\mathrm{V}$, the proposed algorithm is analytically presented in a multi-dimensional Markov chain model and compared with the distributed algorithm in terms of blocking probability, average networks load and average system throughput. Analytical results are validated in this section by comparing them with simulation results. Finally, this paper is concluded in Section VI.

\section{PRoposed Mobility OPTIMIZATION RAT SELECTION APPROACH}

Centralized RAT selection algorithms do not guarantee the required QoS for the admitted calls and reduce the network capacity. Distributed RAT selection algorithms do not consider network preferences and policies. In this section, we presents the intelligent hybrid RAT selection approach for mobility optimization which uses the IEEE P1900.4 Protocol [11] to enable communication between the UTs and the different wireless networks.

Different users can be in different velocity types such as low, medium or high mobility. The proposed intelligent mobility optimization algorithm aims to reduce unnecessary handover. Therefore, low mobility UTs can be allocated to a RAT that has small coverage area such as WLAN; however, high mobility UTs can be allocated to a RAT that has higher coverage area such as WWAN technologies. In addition, RATs policies which include supported services types and load threshold policies need to be considered.

The proposed intelligent hybrid RAT selection approach for mobility optimization will consider the previous factors for each new or VHO call and allocate each call to an optimal RAT depending on the UT requirements and the current RATs status.

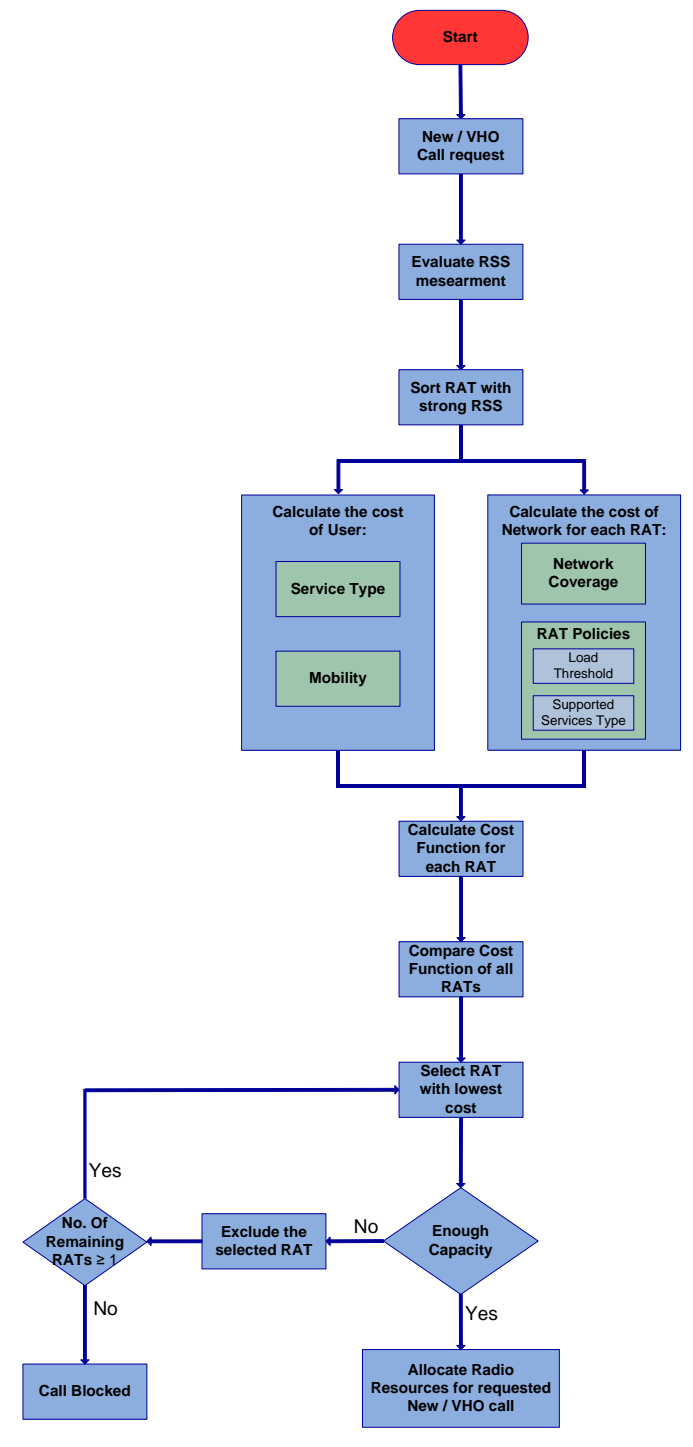

Figure 1. A mobility optimization CRRM approach

The flow chart of the proposed intelligent hybrid RAT selection approach for mobility optimization is presented in Fig. 1. When a new or VHO call is requested, UT will first evaluate the Received Signal Strength (RSS) measurements and makes a list of available RATs that have a strong RSS to accommodate the requested call. Then, the UT will collect information on each RAT using the IEEE P1900.4 Protocol. These information will cover network coverage costs of each RAT and RAT policy attributes such as supported services type and load threshold. A cost will be given for each attribute for each RAT. Other costs will be given for the user attributes, such as: requested service type, user mobility status (low, medium or high). After that, a cost function for each RAT is calculated. Then, a comparison between these costs is made. The RAT with the lowest cost will be allocated for 
the requested call. If the selected RAT does not have enough capacity to allocate the requested call, another RAT will be selected. If none of the available RATs have enough capacity to serve the requested call, the call will be dropped or blocked.

\section{A Cost FunCtion Model}

This section presents the cost function for the allocation of radio resources in the proposed intelligent mobility optimization RAT selection approach for the requested user calls in heterogeneous wireless networks. Calls will be allocated to the RAT that has the lowest cost of allocation. If the selected RAT is unable to serve a call, another RAT will be selected. If none of the RATs are able to serve the call, it will be blocked / dropped. The cost of allocation is calculated by the following equation:

$$
C=C_{N}+C_{U}
$$

where

$\mathrm{C}_{\mathrm{N}}$ is the cost of network.

$\mathrm{C}_{\mathrm{U}}$ is the cost of user.

The cost of network is calculated as follows:

$$
C_{N}=\frac{\sum_{j=1}^{N}\left(W_{j} \times P_{j}\right)}{\sum_{j=1}^{N} W_{j}}
$$

where

$\mathrm{P}_{\mathrm{j}}$ is the parameter related to allocation of resources in the network, such as: network coverage cost $\left(\mathrm{P}_{\mathrm{NC}}\right)$, supported service type cost $\left(\mathrm{P}_{\mathrm{SST}}\right)$ and load threshold cost $\left(\mathrm{P}_{\mathrm{LTH}}\right)$.

$\mathrm{W}_{\mathrm{j}}$ is the factor weight for each network parameter cost.

By adding the above parameters and weight factors, Equation (2) will be:

$$
C_{N}=\frac{W_{N C} \times P_{N C}+W_{S S T} \times P_{S S T}+W_{L T H} \times P_{L T H}}{W_{N C}+W_{S S T}+W_{L T H}}
$$

The cost of user is calculated as follows:

$$
C_{U}=\frac{\sum_{i=1}^{N}\left(W_{i} \times P_{i}\right)}{\sum_{i=1}^{N} W_{i}}
$$

where

$\mathrm{P}_{\mathrm{i}}$ is the parameter related to the usage of resources depending on user status, such as: requested service type cost $\left(\mathrm{P}_{\mathrm{RST}}\right)$ and cost of mobility $\left(\mathrm{P}_{\mathrm{M}}\right)$.

$\mathrm{W}_{\mathrm{i}}$ is the factor weight for each user parameter cost.

By adding the above parameters and weight factors, Equation (4) will be:

$$
C_{U}=\frac{W_{R S T} \times P_{R S T}+W_{M} \times P_{M}}{W_{R S T}+W_{M}}
$$

\section{SIMULATION ENVIRONMENT AND RESUlTS}

In this section, the proposed intelligent mobility optimization algorithm is simulated in a multi-access network scenario. The system model and the simulation results are presented in the following subsections.

\section{A. System Model}

The comparison for the performance of centralized load-balancing, distributed and the proposed intelligent mobility optimization algorithms is simulated in a scenario which is assumed that UTRAN, LTE and WLAN are overlapped in the same geographical area. The area which has coverage for the three RATs is named: hotspot area. The network topology is shown in Fig. 2.

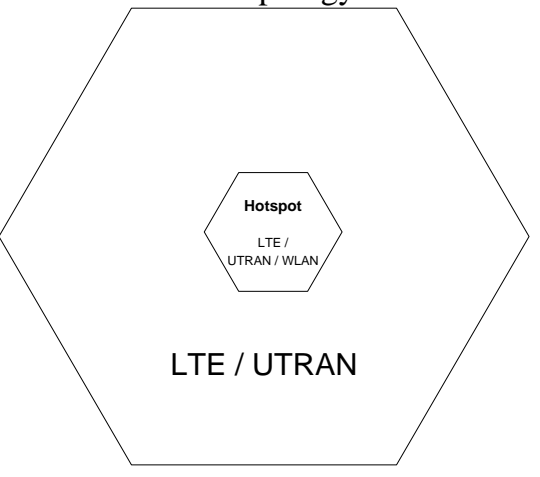

Figure 2. Multi-access cellular network

It is assumed that users arrive and can move inside or outside the hotspot area. Three different service types are considered: voice, data and video calls. Two different video call types are considered: video calls with low resolution and video calls with high resolution (video conference calls). It is assumed that the calls arrivals are generated according to a Poisson process with a mean rate of $\lambda$ [14]. It is also assumed that each call is held for an exponentially distributed call duration time with a mean of $1 / \mu[14,15]$.

In UTRAN, the transmission scheme is based on WCDMA. Voice calls are accepted with a data rate of $12.2 \mathrm{kbps}$. Video calls with low resolution are accepted with a data rate of $48 \mathrm{kbps}$. Video calls with high resolution are accepted with a data rate of $384 \mathrm{kbps}$. Data calls are accepted with a minimum data rate of $64 \mathrm{kbps}$ and it can achieve a maximum data rate of $384 \mathrm{kbps}$. It is assumed that the load threshold factor is 0.8 , WCDMA chip rate is $3.84 \mathrm{Mcps}$, the orthogonality factor is 0.5 and the other cell to own cell interference ratio factor is 0.65 . A list of other parameters and assumptions for the UTRAN network are shown in Table I [16].

The load factors for uplink and downlink in UTRAN are calculated as follows [16]:

$$
\eta_{U L}=(1+i) ? \sum_{j=1}^{N} \frac{1}{1+\frac{W}{\left(E_{b} / N_{o}\right)_{j} ? R_{j} v_{j}}}
$$

where $\mathrm{N}$ is the number of users per cell, $v_{\mathrm{j}}$ is the activity factor of user $j$ at physical layer, $\mathrm{E}_{\mathrm{b}} / \mathrm{N}_{\mathrm{o}}$ is the signal energy per bit divided by noise spectral density, $\mathrm{W}$ is the 
chip rate, $R_{j}$ is the data rate of user $j$ and $i$ is the other cell to own cell interference ratio seen by the base station receiver. The load factor for the downlink is:

$$
\eta_{D L}=\sum_{j=1}^{N} v_{j} \times \frac{\left(E_{b} / N_{o}\right)_{j}}{W / R_{j}} \times\left[\left(1-\alpha_{j}\right)+i_{j}\right]
$$

where $\alpha_{j}$ is the orthogonality of channel of user $j$ and $i_{j}$ is the ratio of other cell to own cell base station received by user $j$.

TABLE I. UTRAN PARAMETERS

\begin{tabular}{|c|c|c|c|c|c|}
\hline Parameter & Voice Call & & $\begin{array}{l}\text { Data } \\
\text { Call }\end{array}$ & $\begin{array}{l}\text { Low } \\
\text { Resolution } \\
\text { Video Call } \\
\end{array}$ & $\begin{array}{l}\text { High } \\
\text { Resolution } \\
\text { Video Call } \\
\end{array}$ \\
\hline $\begin{array}{l}\text { WCDMA } \\
\text { chip rate }\end{array}$ & \multicolumn{5}{|l|}{ 3.84 Mcps } \\
\hline Orthogonality & \multicolumn{5}{|l|}{0.5} \\
\hline $\begin{array}{l}\text { Other Cell to } \\
\text { Own Cell } \\
\text { Interference } \\
\text { Ratio }\end{array}$ & \multicolumn{5}{|l|}{0.65} \\
\hline $\begin{array}{l}\text { Load } \\
\text { Threshold }\end{array}$ & \multicolumn{5}{|l|}{0.8} \\
\hline \multirow{2}{*}{$\begin{array}{l}\text { Activity } \\
\text { Factor }\end{array}$} & Uplink & 0.67 & \multirow{2}{*}{1} & \multirow{2}{*}{1} & \multirow{2}{*}{1} \\
\hline & Downlink & 0.58 & & & \\
\hline $\begin{array}{lr}\text { Signal } & \text { to } \\
\text { Noise } & \text { Ratio } \\
\left(\mathrm{E}_{\mathrm{b}} / \mathrm{N}_{\mathrm{o}}\right) & \\
\end{array}$ & \multicolumn{2}{|l|}{$7.0 \mathrm{db}$} & $\begin{array}{l}5.0 \\
\mathrm{db}\end{array}$ & $4.0 \mathrm{db}$ & $3.2 \mathrm{db}$ \\
\hline Data Rate & \multicolumn{2}{|l|}{$12.2 \mathrm{kbps}$} & $\begin{array}{l}64 \\
\text { kbps }\end{array}$ & $48 \mathrm{kbps}$ & $384 \mathrm{kbps}$ \\
\hline
\end{tabular}

In LTE, the transmission scheme is based on Orthogonal Frequency Division Multiple Access (OFDMA) for downlink and Single Carrier Frequency Division Multiple Access (SC-FDMA) for uplink. The allocated bandwidth is divided into a number of physical Resource Blocks (RBs) in term of frequency, each one has $180 \mathrm{KHz}$. Each RB is divided into 84 Resource Elements (12 subcarriers in terms of frequency $\times 7$ symbols in terms of time), each one has a $15 \mathrm{KHz}$ bandwidth. The load factor for connected user $j$ is calculated as follows:

$$
L_{j}=\frac{R B_{j} \times\left(1+R_{j}\right) \times v_{j}}{f r_{j}}
$$

where

$\mathrm{RB}_{\mathrm{j}}$ is the required number of resource blocks to transmit in one Transmission Time Interval (TTI) for user $j$.

$\mathrm{R}_{\mathrm{j}}$ is the average ratio of retransmission for user $j$.

$v_{\mathrm{j}}$ is the activity factor of user $j$.

$f r{ }_{j}$ is the average TTI where a new packet is transmitted from user $j$.

In WLAN, the transmission scheme is also based on OFDMA. Load of WLAN is calculated by the following equation:

$$
L_{W}=\sum_{j=1}^{N} \frac{R_{j} \times v_{j}}{W_{C}}
$$

where

$\mathrm{N}$ is the number users served in WLAN,

$R_{j}$ is the data rate of user $j$,

$v_{\mathrm{j}}$ is the activity factor of user $j$,

$\mathrm{W}_{\mathrm{C}}$ is the available WLAN capacity.

\section{B. Simulation Performance Results}

The proposed intelligent mobility optimization algorithm is compared with the centralized loadbalancing, and the distributed algorithms in terms of new call blocking probability, VHO call dropping probability and users' satisfactions probability.

The centralized load-balancing algorithm aims to distribute traffic load between all available RATs in a heterogeneous wireless network. Balancing load between all available RATs in a heterogeneous wireless network offers an efficient utilization of the radio resources [1719]. In the centralized load-balancing algorithm, calls are allocated to the RAT that has the minimum load which is based on the decision made by the core network or the base station.

In the distributed algorithm, the RAT selection decision is made by the UT, where the users with high mobility select the RATs that have the highest geographical coverage without considering any of the network factors such as network capacity and supported service type for the selected RAT.

The proposed intelligent mobility optimization algorithm aims to increase users' satisfactions by allocating users with high mobility to a RAT that has high coverage area and at the same time, it aims to improve the efficiently of radio resource utilization by minimizing unnecessary handover. More details on this algorithm are available in Section II.

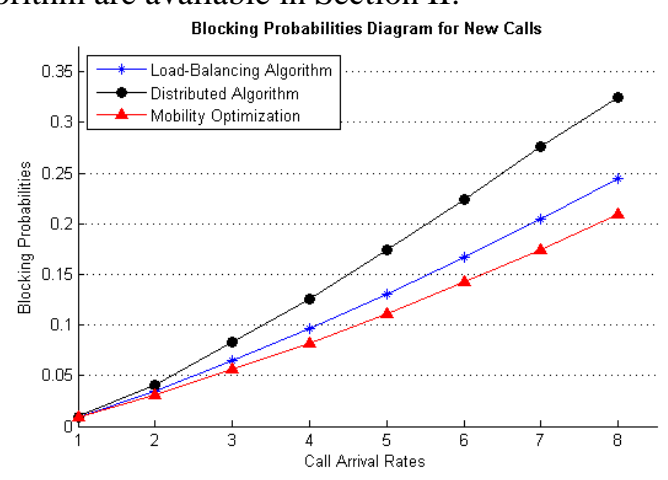

Figure 3. New calls blocking probability

Fig. 3 shows the blocking probability for the three CRRM algorithms. This figure shows that the proposed algorithm outperforms the centralized load-balancing and distributed algorithms in term of blocking probability. The proposed algorithm performs better than the centralized load-balancing algorithm because the loadbalancing algorithm leads to high levels of unnecessary VHO which reduces the overall network capacity. In addition, the network capacity is reduced in centralized load-balancing algorithm as a result of the signaling load and delays introduced by the communication between the network entities during the RAT decision process. 
Distributed algorithm shows the lowest levels of performance when compared to the other two strategies. This is because the distributed algorithm does not consider any of the network factors such as load threshold.

Fig. 4 shows the dropping probability for the three CRRM algorithms. It can be seen that the proposed algorithm performs better than the centralized loadbalancing algorithm as a result of the reduced network capacity introduced by the high unnecessary VHO and the increased signaling load produced during the communication between the network entities in the RAT decision process. The proposed algorithm outperforms also the distributed algorithm which is the worst one in term of dropping probability.

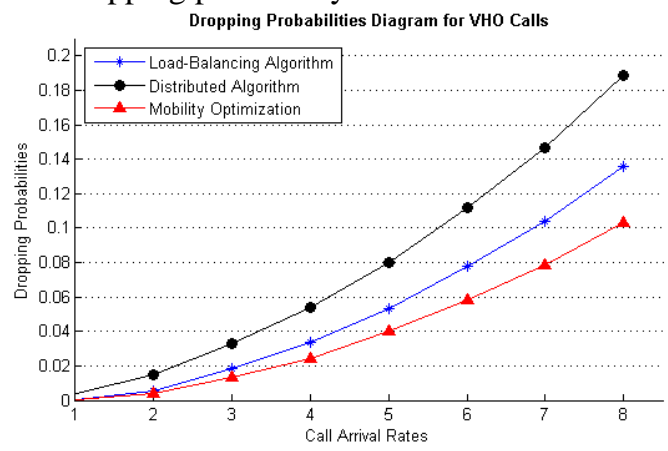

Figure 4. VHO calls dropping probability

Fig. 5 illustrates the users' satisfactions probability for the three CRRM algorithms. Simulation results show that the proposed algorithm and the distributed algorithm perform better than the centralized load-balancing algorithm. This is because, both proposed and distributed algorithms consider users' preferences which increase their satisfactions. Distributed and proposed algorithms have similar performance when traffic level is low. However, when the traffic becomes high, the proposed algorithm outperforms the distributed algorithm in term of users' satisfactions probability. This is because that the distributed algorithm does not consider any of the network factors such as load threshold.

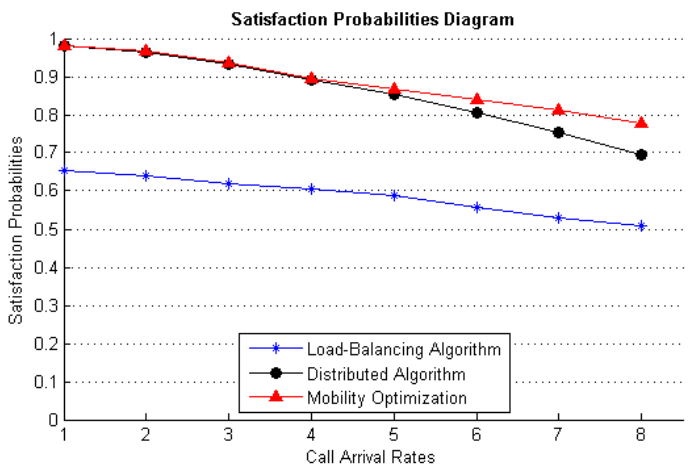

Figure 5. Users' satisfactions probability

Fig. 6 shows the average satisfaction increasing percentages for the proposed algorithm compared with the centralized load-balancing and the distributed algorithms. Average satisfaction increasing percentages are calculated by subtracting the users' satisfactions probability of the load-balancing and distributed algorithms from the users' satisfactions probability of the proposed algorithm. This figure shows that the users' satisfactions have been increased around 33\% against the load-balancing algorithm when traffic load is low and around $27 \%$ when traffic load is high. It also shows that the users' satisfactions percentages for the proposed algorithm are similar to the distributed algorithm when traffic load is low; however, users' satisfactions have been increased around 9\% when traffic load is high.

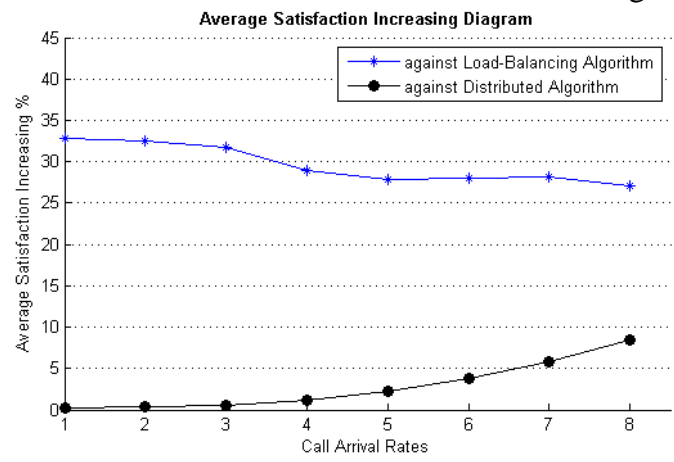

Figure 6. Average satisfaction increasing percentages

\section{Multi-Dimensional Markov Chain Model}

In this section, a multi-dimensional Markov chain model for the proposed intelligent mobility optimization CRRM approach is presented. Markov model has been used by [20-24] to analytically model RAT selection algorithms. A heterogeneous wireless network which assumes that UTRAN, LTE and WLAN are overlapped in the same geographical area is considered for the Markovian model. It is assumed that LTE has higher coverage than UTRAN. It is also assumed that users arrive and can move inside or outside the hotspot area. Two different service types are considered: real time (RT: voice calls) and non real time (NRT: data calls) services where $\mathrm{P}_{\mathrm{v}}$ is the probability of users being voice call and $\mathrm{P}_{\mathrm{d}}$ is the probability of users being data call. $\mathrm{P}_{\text {user_in }}$ and $\mathrm{P}_{\text {user_out }}$ are the probability of users being inside and outside the hotspot area respectively; therefore:

$$
\mathrm{P}_{\text {user_in }}+\mathrm{P}_{\text {user_out }}=1
$$

Let $S_{(i, j, k, l, m, n)}$ represents a state of the system where $i$ is the number of voice call in UTRAN, $j$ is the number of data call in UTRAN, $k$ is the number of voice call in LTE, $l$ is the number of data call in LTE, $m$ is the number of voice call in WLAN, and $n$ is the number of data call in WLAN. Let $S$ denote the set of all possible states within UTRAN, LTE and WLAN as follows:

$$
S=\left\{S_{(i, j, k, l, m, n)} \forall i, j, k, l, m, n\right\}
$$

The transitions between states take places due to the new call arrival rates or VHO call arrival rates. In this model, it is assumed that $\lambda_{v}^{U}$ and $\lambda_{d}^{U}$ are the new call arrival rates for voice and data calls in UTRAN respectively. $\lambda_{v}^{L}$ and $\lambda_{d}^{L}$ are the new call arrival rates for voice and data calls in LTE respectively. $\lambda_{v}^{W}$ and $\lambda_{d}^{W}$ are 
the new call arrival rates for voice and data calls in WLAN respectively. It is also assumed that $\lambda_{v}^{U L}$ and $\lambda_{d}^{U L}$ are the VHO call arrival rates for voice and data calls from UTRAN to LTE respectively. $\lambda_{v}^{U W}$ and $\lambda_{d}^{U W}$ are the VHO call arrival rates for voice and data calls from UTRAN to WLAN respectively. $\lambda_{v}^{L U}$ and $\lambda_{d}^{L U}$ are the VHO call arrival rates for voice and data calls from LTE to UTRAN respectively. $\lambda_{v}^{L W}$ and $\lambda_{d}^{L W}$ are the VHO call arrival rates for voice and data calls from LTE to WLAN respectively. $\lambda_{v}^{W U}$ and $\lambda_{d}^{W U}$ are the VHO call arrival rates for voice and data calls from WLAN to UTRAN respectively. $\lambda_{v}^{W L}$ and $\lambda_{d}^{W L}$ are the VHO call arrival rates for voice and data calls from WLAN to LTE respectively.

The proposed intelligent hybrid mobility optimization algorithm is compared with the distributed mobility optimization algorithm in the heterogeneous wireless network. These algorithms are analytically shown in the following subsections.

\section{A. Intelligent Hybrid Mobility Optimization Algorithm}

The proposed intelligent hybrid mobility optimization algorithm aims to allocate users with high mobility to the RAT that has higher coverage area with assistance from the network. This will minimize unnecessary VHO. Initial RAT and VHO RAT selection of the proposed intelligent mobility optimization algorithm in the assumed system model is presented in Fig. 7 and Fig. 8.

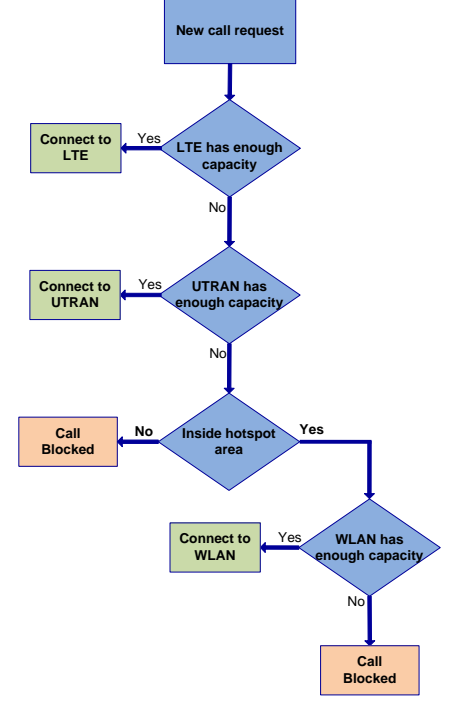

Figure 7. Initial RAT selection

According to this algorithm, the new and VHO call arrival rates are calculated. In order to determine whether if new or VHO call will be allocated to UTRAN, LTE or WLAN in the proposed mobility optimization algorithm, the following indicator functions have been defined:

$$
\begin{gathered}
\alpha_{(i, j, k, l, m, n)}= \begin{cases}1 & \text { if } L_{L}>1 \quad \& \quad L_{U} \leq 1 \quad \text { or } \\
0 & \text { othervise }\end{cases} \\
\beta_{(i, j, k, l, m, n)}=\left\{\begin{array}{lll}
1 & \text { if } L_{L} \leq 1 \\
0 & \text { othervise }
\end{array}\right.
\end{gathered}
$$

$$
\delta_{(i, j, k, l, m, n)}=\left\{\begin{array}{l}
1 \quad \text { if } L_{U}>1 \& L_{L}>1 \& L_{W} \leq 1 \text { or } \\
0 \quad \text { othervise }
\end{array}\right.
$$

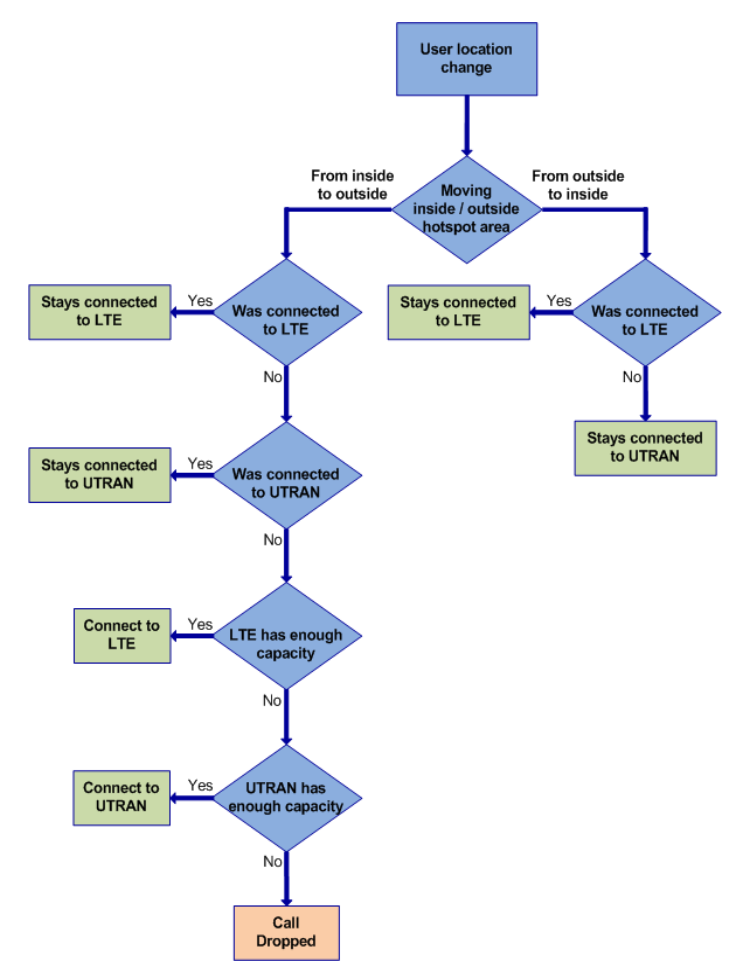

Figure 8. VHO RAT selection

The new voice call and data call arrival rate in UTRAN for the proposed mobility optimization algorithm is calculated by the following equations:

$$
\begin{aligned}
& \lambda_{v(i, j, k, l, m, n)}^{U}=\lambda_{N} \times P_{v} \times\left(P_{u_{\text {user_in }}} \times \alpha_{(i, j, k, l, m, n)}\right. \\
& \left.+P_{\text {user_out }} \times \alpha_{(i, j, k, l, m, n)}\right) \\
& \lambda_{d(i, j, k, l, m, n)}^{U}=\lambda_{N} \times P_{d} \times\left(P_{\text {user_in }} \times \alpha_{(i, j, k, l, m, n)}\right. \\
& \left.+P_{\text {user_out }} \times \alpha_{(i, j, k, l, m, n)}\right)
\end{aligned}
$$

Using Equation (10), the new voice call and data call arrival rate in UTRAN will be:

$$
\begin{aligned}
& \lambda_{v(i, j, k, l, m, n)}^{U}=\lambda_{N} \times P_{v} \times \alpha_{(i, j, k, l, m, n)} \\
& \lambda_{d(i, j, k, l, m, n)}^{U}=\lambda_{N} \times P_{d} \times \alpha_{(i, j, k, l, m, n)}
\end{aligned}
$$

The new voice call and data call arrival rate in LTE for the proposed mobility optimization algorithm is calculated by the following equations:

$$
\begin{aligned}
& \lambda_{v(i, j, k, l, m, n)}^{L}=\lambda_{N} \times P_{v} \times\left(P_{u s e r_{-} i n} \times \beta_{(i, j, k, l, m, n)}\right. \\
& \left.+P_{\text {user_out }} \times \beta_{(i, j, k, l, m, n)}\right) \\
& \lambda_{d(i, j, k, l, m, n)}^{L}=\lambda_{N} \times P_{d} \times\left(P_{u s e r_{-} i n} \times \beta_{(i, j, k, l, m, n)}\right. \\
& \left.+P_{\text {user_out }} \times \beta_{(i, j, k, l, m, n)}\right)
\end{aligned}
$$

Using Equation (10), the new voice call and data call arrival rate in LTE will be: 


$$
\begin{aligned}
& \lambda_{v(i, j, k, l, m, n)}^{L}=\lambda_{N} \times P_{v} \times \beta_{(i, j, k, l, m, n)} \\
& \lambda_{d(i, j, k, l, m, n)}^{L}=\lambda_{N} \times P_{d} \times \beta_{(i, j, k, l, m, n)}
\end{aligned}
$$

The new voice call and data call arrival rate in WLAN for the proposed mobility optimization algorithm is calculated by the following equations:

$$
\begin{aligned}
& \lambda_{v(i, j, k, l, m, n)}^{W}=\lambda_{N} \times P_{v} \times P_{\text {user_in }} \times \delta_{(i, j, k, l, m, n)} \\
& \lambda_{d(i, j, k, l, m, n)}^{W}=\lambda_{N} \times P_{d} \times P_{\text {user_in }} \times \delta_{(i, j, k, l, m, n)}
\end{aligned}
$$

The VHO arrival rate for voice calls and data calls from WLAN to UTRAN for the proposed mobility optimization algorithm is calculated by the following equations:

$$
\begin{aligned}
& \lambda_{v(i, j, k, l, m, n)}^{W U}=m \times P_{\text {user_in }} \times P_{\text {user_exit }} \times \alpha_{(i, j, k, l, m, n)} \\
& \lambda_{d(i, j, k, l, m, n)}^{W U}=n \times P_{\text {user_in }} \times P_{\text {user_exit }} \times \alpha_{(i, j, k, l, m, n)}
\end{aligned}
$$

The VHO arrival rate for voice calls and data calls from WLAN to LTE for the proposed mobility optimization algorithm is calculated by the following equations:

$$
\begin{aligned}
& \lambda_{v(i, j, k, l, m, n)}^{W L}=m \times P_{\text {user_in }_{-}} \times P_{u s e r_{-} \text {exit }} \times \beta_{(i, j, k, l, m, n)} \\
& \lambda_{d(i, j, k, l, m, n)}^{W L}=n \times P_{\text {user_in }_{\text {_in }}} \times P_{u s e r_{\text {_exit }}} \times \beta_{(i, j, k, l, m, n)}
\end{aligned}
$$

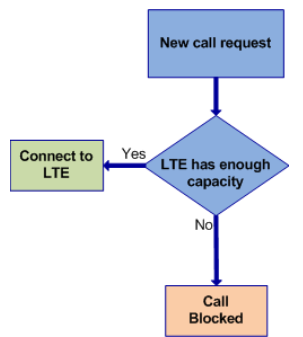

Figure 9. Initial RAT selection

As the proposed mobility optimization algorithm aims to minimize unnecessary $\mathrm{VHO}$, therefore the $\mathrm{VHO}$ arrival rate for voice and data calls from UTRAN to WLAN, from LTE to WLAN, from LTE to UTRAN and from UTRAN to LTE are equal to zero as follows:

$$
\begin{aligned}
& \lambda_{v(i, j, k, l, m, n)}^{U W}=\lambda_{d(i, j, k, l, m, n)}^{U W}=0 \\
& \lambda_{v(i, j, k, l, m, n)}^{L W}=\lambda_{d(i, j, k, l, m, n)}^{L W}=0 \\
& \lambda_{v(i, j, k, l, m, n)}^{L U}=\lambda_{d(i, j, k, l, m, n)}^{L U}=0 \\
& \lambda_{v(i, j, k, l, m, n)}^{U L}=\lambda_{d(i, j, k, l, m, n)}^{U L}=0
\end{aligned}
$$

\section{B. Distributed Mobility Optimization Algorithm}

The distributed mobility optimization algorithm allocates users with high mobility to the RAT that has higher coverage area without considering any of the network preferences and policies. If the selected RAT is unable to allocate the requested new / VHO call, it will be blocked / dropped. Initial RAT and VHO RAT selection of the distributed mobility optimization algorithm in the assumed system model is presented in Fig. 9 and Fig. 10.

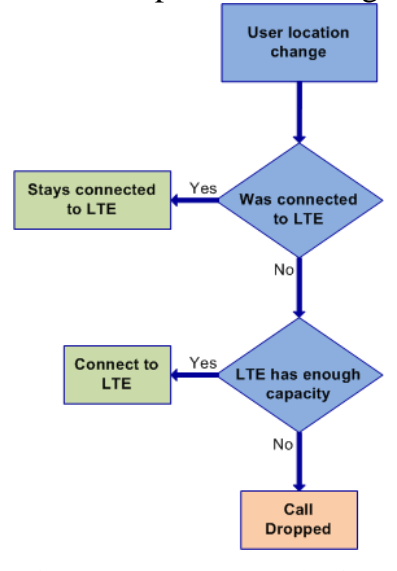

Figure 10. VHO RAT selection

According to this algorithm, the new and VHO call arrival rates are calculated. In order to determine whether if new or VHO call will be allocated to UTRAN, LTE or WLAN in the distributed mobility optimization algorithm, the following indicator function has been defined:

$$
\beta_{(i, j, k, l, m, n)}=\left\{\begin{array}{l}
1 \quad \text { if } L_{L} \leq 1 \quad \text { or } \\
0 \quad \text { othervise }
\end{array}\right.
$$

The new voice call arrival rate in LTE for the distributed mobility optimization algorithm is calculated by the following equation:

$$
\lambda_{v(i, j, k, l, m, n)}^{L}=\lambda_{N} \times P_{v} \times \beta_{(i, j, k, l, m, n)}
$$

The new data call arrival rate in LTE for the distributed mobility optimization algorithm is calculated by the following equation:

$$
\lambda_{d(i, j, k, l, m, n)}^{L}=\lambda_{N} \times P_{d} \times \beta_{(i, j, k, l, m, n)}
$$

As the distributed mobility optimization algorithm allocates users with high mobility to LTE only, therefore the new voice and data call arrival rates in UTRAN and WLAN for the distributed mobility optimization algorithm are equal to zero as follows:

$$
\begin{aligned}
& \lambda_{v(i, j, k, l, m, n)}^{U}=\lambda_{d(i, j, k, l, m, n)}^{U}=0 \\
& \lambda_{v(i, j, k, l, m, n)}^{W}=\lambda_{d(i, j, k, l, m, n)}^{W}=0
\end{aligned}
$$

The VHO arrival rate for voice calls and data calls from WLAN to LTE for the distributed mobility optimization algorithm is calculated by the following equations:

$$
\begin{aligned}
& \lambda_{v(i, j, k, l, m, n)}^{W L}=m \times \beta_{(i, j, k, l, m, n)} \\
& \lambda_{d(i, j, k, l, m, n)}^{W L}=n \times \beta_{(i, j, k, l, m, n)}
\end{aligned}
$$


As $m=0$ and $n=0$, this is because the distributed mobility optimization algorithm allocates users with high mobility to LTE only, therefore:

$$
\begin{aligned}
& \lambda_{v(i, j, k, l, m, n)}^{W L}=0 \\
& \lambda_{d(i, j, k, l, m, n)}^{W L}=0
\end{aligned}
$$

The VHO arrival rate for voice calls and data calls from UTRAN to LTE for the distributed mobility optimization algorithm is calculated by the following equations:

$$
\begin{aligned}
& \lambda_{v(i, j, k, l, m, n)}^{U L}=i \times \beta_{(i, j, k, l, m, n)} \\
& \lambda_{d(i, j, k, l, m, n)}^{U L}=j \times \beta_{(i, j, k, l, m, n)}
\end{aligned}
$$

As $i=0$ and $j=0$, this is because the distributed mobility optimization algorithm allocates users with high mobility to LTE only, therefore:

$$
\begin{aligned}
& \lambda_{v(i, j, k, l, m, n)}^{U L}=0 \\
& \lambda_{d(i, j, k, l, m, n)}^{U L}=0
\end{aligned}
$$

As the distributed mobility optimization algorithm allocates users with high mobility to LTE only, therefore the VHO arrival rate for voice and data calls from WLAN to UTRAN, from UTRAN to WLAN, from LTE to WLAN and from LTE to UTRAN are equal to zero as follows:

$$
\begin{aligned}
& \lambda_{v(i, j, k, l, m, n)}^{W U}=\lambda_{d(i, j, k, l, m, n)}^{W U}=0 \\
& \lambda_{v(i, j, k, l, m, n)}^{U W}=\lambda_{d(i, j, k, l, m, n)}^{U W}=0 \\
& \lambda_{v(i, j, k, l, m, n)}^{L W}=\lambda_{d(i, j, k, l, m, n)}^{L W}=0 \\
& \lambda_{v(i, j, k, l, m, n)}^{L U}=\lambda_{d(i, j, k, l, m, n)}^{L U}=0
\end{aligned}
$$

\section{Analytical Performance Results}

The intelligent hybrid mobility optimization algorithm is compared with the distributed mobility optimization algorithm in terms of blocking probabilities, average network load and average system throughput.

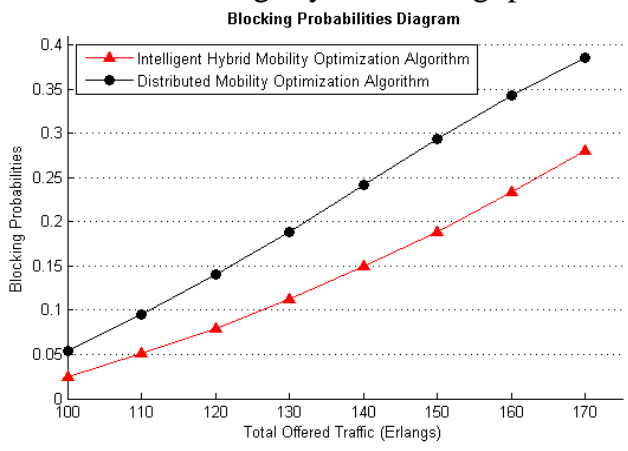

Figure 11. Blocking probabilities
The blocking probabilities for the proposed intelligent hybrid mobility optimization algorithm and the distributed mobility optimization algorithm are presented in Fig. 11. This figure shows that the proposed intelligent mobility optimization algorithm outperforms the distributed algorithm in term of blocking probabilities. This is because the distributed mobility optimization algorithm allocates users with high mobility to the RAT that has higher coverage area without considering any of the network factors and policies such as load threshold.

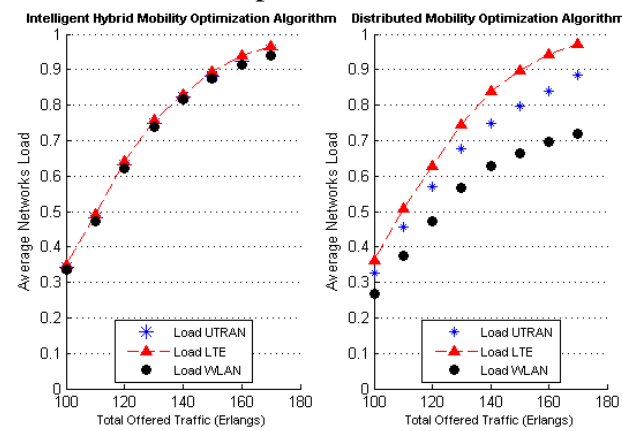

Figure 12. Average networks load

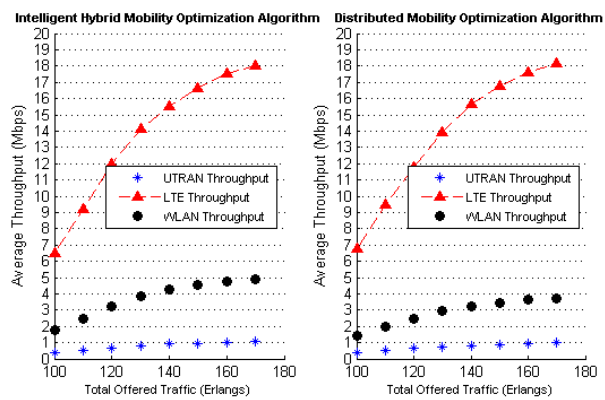

Figure 13. Average system throughput

Fig. 12 and Fig. 13 show the average network load and the average system throughput in each RAT for the proposed and distributed mobility optimization algorithms. It can be seen that the proposed intelligent mobility optimization algorithm distributes the traffic load among all available RATs. However, calls traffics are allocated to LTE more than to UTRAN and WLAN in the distributed algorithm. This is because the distributed mobility optimization algorithm allocates users with high mobility to LTE only. As a result, the proposed mobility optimization algorithm performs better than the distributed algorithm in term of average system throughput.

\section{Validation of Analytical Performance Results}

In order to validate the results obtained for the proposed mobility optimization algorithm in the multidimensional Markov model, a simulation model is developed again using the same system model and same assumptions implemented in the Markovian model. The simulation and analytical results are compared in terms blocking probabilities, average network load and average system throughput.

Fig. 14, Fig. 15 and Fig. 16 compares the blocking probabilities, average network load and average system throughput results obtained via the simulation with the 
results obtained via the Markovian model for the proposed intelligent mobility optimization algorithm. It can be seen clearly that both simulation and analytical results are matching. Therefore, the results obtained for the proposed intelligent mobility optimization algorithm are valid.

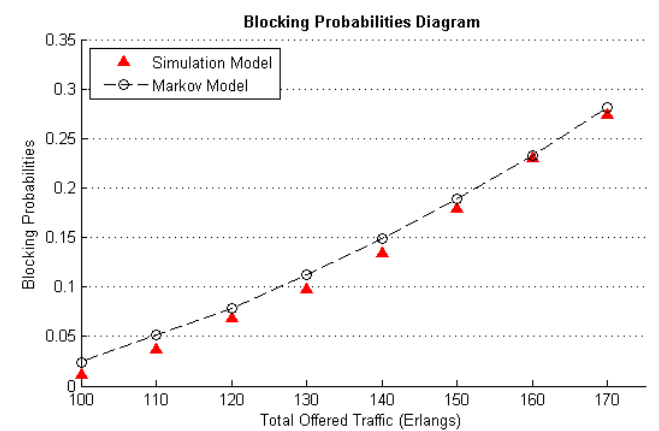

Figure 14. Blocking probabilities for the proposed intelligent mobility optimization algorithm

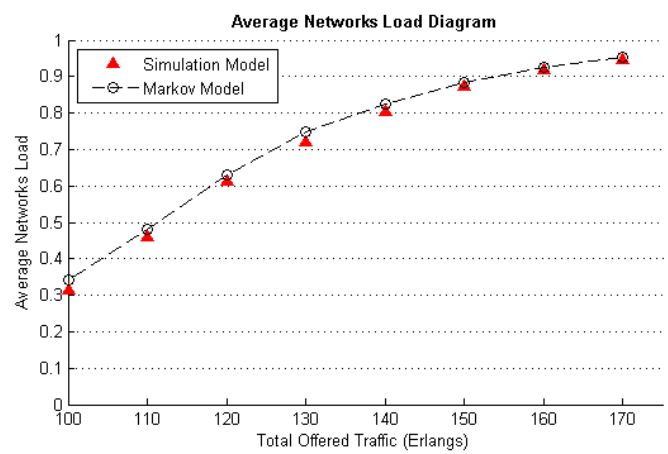

Figure 15. Average networks load for the proposed intelligent mobility optimization algorithm

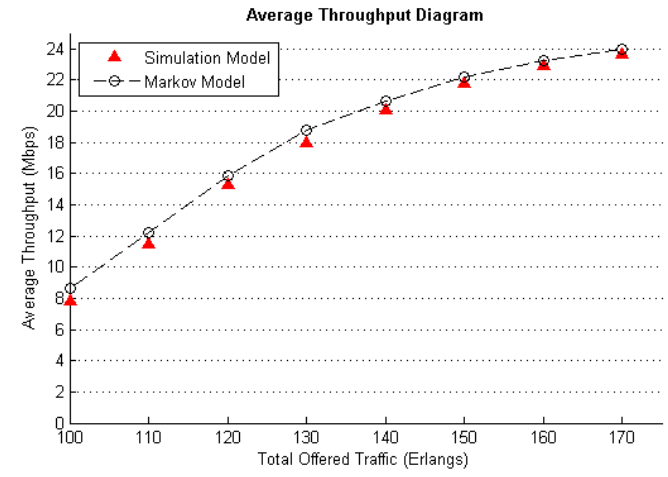

Figure 16. Average system throughput for the proposed intelligent mobility optimization algorithm

\section{CONCLUSIONS}

An intelligent RAT selection approach is necessary for more efficient utilization of radio resources in heterogeneous wireless networks and to increase users' satisfactions and guarantee the required QoS for allocated calls. This paper presents the proposed mobility optimization RAT selection approach and proposes an analytical presentation of this approach in a multidimensional Markov chain model. The proposed algorithm has been compared with the centralized loadbalancing and distributed algorithms in terms of new calls blocking probability, VHO calls dropping probability, users' satisfactions probability, average networks load and average system throughput. Simulation and analytical results show that the proposed algorithm is more efficient than centralized and distributed algorithms.

\section{ACKNOWLEDGMENT}

This work is sponsored by the Centre for Real-Time Information Networks (CRIN) in the Faculty of Engineering \& Information Technology at the University of Technology, Sydney (UTS).

\section{REFERENCES}

[1] Motorola, "Technical White Paper: Long Term Evolution (LTE): A Technical Overview," 2010

[2] V. K. Garg, Wireless Communications and Networking, Morgan Kaufmann Publishers, San Francisco, CA, USA 2007.

[3] P. Nicopolitidis, M. S. Obaidat, G. I. Papadimitriou, and A. S. Pomportsis, Wireless Networks, John Wiley \& Sons Ltd, Chichester, UK 2003.

[4] J. Schiller, Mobile Communications, 2nd Edition, Addison Wesley, Harlow UK 2003.

[5] A. AL Sabbagh, R. Braun and M. Abolhasan, "Centralized and Distributed CRRM in Heterogeneous Wireless Networks", in Advanced Methods and Applications in Computational Intelligence, Topics in Intelligent Engineering and Informatics, vol. 6, Springer International Publishing, Switzerland 2014, pp. 299-314.

[6] A. AL Sabbagh, R. Braun and M. Abolhasan, "A Comprehensive Survey on RAT Selection Algorithms for Heterogeneous Networks," World Academy of Science, Engineering and Technology (WASET), no. 73, January 2011, pp. 141-145.

[7] A. AL Sabbagh, R. Braun and M. Abolhasan, "A Comprehensive Survey on RAT Selection Algorithms for Beyond 3G Networks," in International Conference on Communications, Networking and Mobile Computing (ICCNMC 2011), Dubai, UAE, January 25-27, 2011, pp. 834-838.

[8] E. Adamopoulou, K. Demestichas, A. Koutsorodi and M. Theologou, "Intelligent Access Network Selection in Heterogeneous Networks," in 2nd International Symposium on Wireless Communication Systems 2005 (ISWCS'05), Siena, Italy, September 5-7, 2005, pp. 279283.

[9] F. Bari and V. C. M. Leung, "Automated Network Selection in a Heterogeneous Wireless Network Environment," IEEE Network, vol. 21, no. 1, January February 2007, pp. 34-40.

[10] Q. T. Nguyen-Vuong, N. Agoulmine and Y. GhamriDoudane, "Terminal-Controlled Mobility Management in Heterogeneous Wireless Networks," IEEE Communications Magazine, vol. 45, no. 4, April 2007, pp. 122-129.

[11] S. Buljore, et al., "IEEE P1900.4 System Overview on Architecture and Enablers for Optimised Radio and Spectrum resource usage," in 2008 IEEE Symposium on New Frontiers in Dynamic Spectrum Access Networks, Chicago, Illinois, USA, October 14-17, 2008.

[12] A. AL Sabbagh, R. Braun and M. Abolhasan, "A Mobility Optimization CRRM Approach for Next Generation Wireless Networks," International Conference on 
Computer and Information Sciences (ICCIS 2012), Kuala Lumpur, Malaysia, June 12-14, 2012, pp. 609-613.

[13] A. AL Sabbagh, "A Hybrid Radio Resource Allocation Method for Multiple Wireless Access Networks," Australian Innovation Patent AU2013100801, July 2013.

[14] ETSI Technical Report, "Universal Mobile Telecommunications System (UMTS), Selection procedures for the choice of radio transmission technologies of the UMTS," UMTS 30.03 version 3.2.0, March 1998.

[15] Y. Fang, I. Chlamtac and Y. B. Lin, "Modeling PCS Networks Under General Call Holding Time and Cell Residence Time Distributions," IEEE/ACM Transactions on Networking, vol. 5, no. 6, Dec. 1997, pp. 893-906.

[16] H. Holma and A. Toskala, WCDMA for UMTS - HSPA Evolution and LTE, fourth Edition, John Wiley \& Sons Ltd, Chichester, UK 2007.

[17] A. Tolli and P. Hakalin, "Adaptive Load Balancing Between Multiple Cell Layers," in 2002 IEEE 56th Vehicular Technology Conference (VTC 2002), vol. 3, Vancouver, Canada, September 24-28, 2002, pp. 16911695.

[18] K. H. Suleiman, H. A. Chan and M. E. Dlodlo, "Load Balancing in the Call Admission Control of Heterogeneous Wireless Networks," in International Conference on Communications and Mobile Computing (IWCMC 2006), Vancouver, British Columbia, Canada, July 3-6, 2006, pp. 245-250.

[19] A. Pillekeit, F. Derakhshan, E. Jugl and A. Mitschele-Thiel, "Force-Based Load Balancing in Co-located UMTS/GSM Networks," in 2004 IEEE 60th Vehicular Technology Conference (VTC 2004), vol. 6, Los Angeles, California, USA, September 26-29, 2004, pp. 4402-4406.

[20] A. AL Sabbagh, "A Markov Chain Model for LoadBalancing Based and Service Based RAT Selection Algorithms in Heterogeneous Networks," World Academy of Science, Engineering and Technology (WASET), no. 73, January 2011, pp. 146-152.

[21] A. AL Sabbagh and R. Braun, "A Markovian Model for Load-Balancing Based and Service Based Algorithms in B3G Networks," in International Conference on Communications, Networking and Mobile Computing (ICCNMC 2011), Dubai, UAE, January 25-27, 2011, pp. 827-833.

[22] A. Hasib and A. O. Fapojuwo, "Analysis of Common Radio Resource Management Scheme for End-to-End QoS Support in Multiservice Heterogeneous Wireless Networks," IEEE Transactions on Vehicular Technology, vol. 57, no. 4, July 2008, pp. 2426-2439.

[23] X. Gelabert, J. Perez-Romero, O. Sallent and R. Agusti, “A 4-Dimensional Markov Model for the Evaluation of Radio Access Technology Selection Strategies in Multiservice Scenarios," in 64th IEEE Vehicular Technology Conference (VTC 2006), Montreal, Canada, September 2528, 2006.

[24] H. ElBadawy, "Modeling and Analysis for Heterogeneous Wireless Networks by Using of Multi-Dimensional Markov Models," in International Conference on Computer and Communication Engineering 2008 (ICCCE'08), Kuala Lumpur, Malaysia, May 13-15, 2008, pp. 1116-1120.

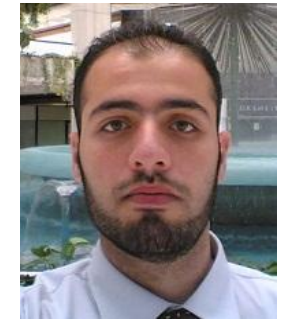

Abdallah AL Sabbagh received his B.Sc. in Information Technology and Computing (Hons) from the Arab Open University, Lebanon and the Open University, UK (2006) and a Master of Engineering Studies (MES) in Telecommunication Networks from the Faculty of Engineering at the University of Technology, Sydney (2010). He is currently with the School of Computing and Communications, and Centre for Real-Time Information Networks (CRIN) at the University of Technology, Sydney (UTS). His recent research is the Radio Resource Management (RRM), Admission Control, Vertical Handover (VHO) and packet scheduling for Next Generation Wireless Networks (NGWN). Abdallah is member of the Centre for Real-time Information Networks (CRIN) and Engineers Australia.

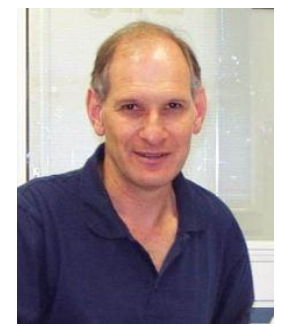

Robin Braun received his B.Sc (Hons) from Brighton University (UK), and his M.Sc and Ph.D from the University of Cape Town. He holds the Chair of Telecommunications Engineering in the Faculty of Engineering and Information Technology of the University of Technology, Sydney, Australia. He is an executive member of the Centre for Real Time Information Networks (CRIN) at the University of Technology, Sydney (UTS). Prof. Braun was a member of staff of the Department of Electrical Engineering of the University of Cape Town from 1986 to 1998 . He was the founder, and Director of the Digital Radio Research Group at the University of Cape Town, which supervised over 50 research degree candidates in the years that he was attached to it. Prof. Braun is currently a Senior Member of the Institute of Electrical and Electronic Engineers of the United States (IEEE).

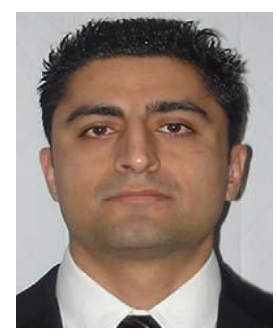

Mehran Abolhasan completed his B.E in Computer Engineering and Ph.D. in Telecommunications on 1999 and 2003 respectively at the University of Wollongong. From July 2003, he joined the Smart Internet Technology CRC and Office of Information and Communication Technology within the Department of Commerce in NSW, where he proposed a major status report outlining strategies and new projects to improve the communications infrastructure between the NSW Emergency Services Organisations. In July 2004, he joined the Desert knowledge CRC and Telecommunication and IT Research Institute to work on a joint project called the Sparse Ad hoc network for Deserts project (Also known as the SAND project). Dr. Abolhasan is currently a Senior Lecturer at the School of Computing and Communications within the faculty of Engineering and IT (FEIT) at the University of Technology Sydney (UTS). He has authored over 80 international publications and has won over one million dollars in research funding over the past 5 years. His Current research Interests are in Wireless Mesh, 4th Generation Cooperative Networks and Sensor networks. Dr. Abolhasan is currently a Senior Member of the Institute of Electrical and Electronic Engineers of the United States (IEEE). 